\title{
Electronic states tuning of InAs self-assembled quantum dots
}

\author{
J. M. García, ${ }^{\text {a) }}$ T. Mankad, P. O. Holtz, P. J. Wellman, and P. M. Petroff ${ }^{\text {b) }}$ \\ Department of Materials and QUEST, University of California, Santa Barbara, California 93106
}

(Received 16 December 1997; accepted for publication 14 April 1998)

\begin{abstract}
We demonstrate the dimensional tuning of InAs self-assembled quantum dots (QDs) by changing the growth kinetics during the capping of InAs islands with GaAs. Modifying the growth sequence during the capping of InAs islands, allows us to tune the thickness and lateral dimensions of the QDs while keeping the wetting layer thickness constant. Using the same method but embedding the tuned InAs islands into AlAs layers allows to further blueshift the photoluminescence emission to higher energies while keeping the wetting layer thickness constant. The main process responsible for the QDs size modification is consistent with a kinetically controlled materials redistribution of the InAs islands that minimizes the energy of the epitaxial layers at the start up of the GaAs capping deposition. (C) 1998 American Institute of Physics. [S0003-6951(98)02024-5]
\end{abstract}

With the steady progress on the understanding of selfassembled quantum dots (QDs) ${ }^{1-3}$ physical and electronic properties, more device applications are currently under investigation. QDs lasers, ${ }^{4}$ detectors, ${ }^{5}$ or charge storage devices ${ }^{6}$ have been reported. More than often, to fully achieve the device potential has remained elusive because of the lack of control over the dimensions of the self-assembled QDs. Here, we propose a method which allows to tune the QDs sizes in an easy and reproducible fashion for the InAs/ GaAs QDs system while keeping the wetting layer (WL) thickness constant to a minimum $(\sim 1.7 \mathrm{ML})$. This could be a desirable characteristic in some devices where the quantum well $(\mathrm{QW})$ like characteristics of the WL could adversely affect the device.

Previous studies have shown that by changing the QDs compositions, ${ }^{7}$ e.g., using AlInAs QDs imbedded in AlGaAs, it is also possible to tune their photoluminescence (PL) wavelength emission. However, this was achieved at the expense of a thicker WL $(\approx 22 \AA)$ since the misfit strain of the AlInAs/AlGaAs structures is smaller than that of the InAs/ GaAs QDs system. Post-growth annealing $\left(900{ }^{\circ} \mathrm{C}\right)$ of InAs/ GaAs QDs has been shown to also blueshift the PL emission. ${ }^{8}$ This effect was attributed to compositional interdiffusion between the islands and the cladding film. Since the electronic properties of the QDs, ${ }^{1-3}$ are sensitive to their shape, size and composition, ${ }^{9-12}$ it is not surprising that different growth conditions (and therefore different growth kinetics) yield different electronic structures. We have already shown that during the overgrowth of InAs islands with GaAs, diffusion and intermixing ${ }^{13}$ with the top cladding GaAs epitaxial layer takes place and produce large changes in the original island sizes and shapes. In this letter we propose to use this effect for tuning the energy levels of the QDs.

To demonstrate the possibility of tuning the wavelength of the dots we have grown by molecular bean epitaxy five different samples on semi-insulating $\operatorname{GaAs}(100)$ under 1

\footnotetext{
${ }^{a)}$ Electronic mail: jorge.garcia@uam.es

${ }^{b)}$ Electronic mail: petroff@engineering.ucsb.edu
}

$\times 10^{-5}$ Torr Arsenic $\left(\mathrm{As}_{2}\right)$ pressure. In all cases a GaAs buffer layer is prepared with a short period superlattice (40 $\times 20 \AA / 20 \AA \mathrm{AlAs} / \mathrm{GaAs}$ ) at a growth temperature of $T_{g}$ $=630{ }^{\circ} \mathrm{C}$. The dots are formed by depositing $1.7 \operatorname{InAs}$ monolayers at $530{ }^{\circ} \mathrm{C}$. A first growth interruption of $30 \mathrm{~s}$ is used to narrow down the size distribution. Then the dots are covered at $530{ }^{\circ} \mathrm{C}$ with a thin $\mathrm{GaAs}$ cap of thickness $t_{\text {cap }}$ adjusted between 10 and $50 \AA$ followed by a second $30 \mathrm{~s}$ growth interruption and a final capping with GaAs or AlAs.

Samples A, B, and C are metal insulator semiconductor field effect transistor (MISFET) structures in which the InAs dots imbedded in GaAs have been grown using this new growth technique with $t_{\text {cap }}=50,30$, and $10 \AA$, where a $200 \AA$ heavily $\left(50 \times 10^{-18} \mathrm{~cm}^{-3}\right)$ Si-doped GaAs layer $250 \AA$ below the dots acts as a back contact. The QDs layer in sample $\mathrm{D}$ is grown on the undoped GaAs buffer layer without the $\mathrm{Si}^{+}$back contact and with $t_{\text {cap }}=10 \AA$ (similar to sample C) and covered with thick GaAs (>200 A). Sample E includes five layers of QDs grown with continuous rotation in which the dots are embedded in AlAs (see inset in Fig. 3). The first four layers $\left(t_{\text {cap }}=10,20,30,40 \AA\right)$ are grown repeating four times the following sequence (see insert in Fig. 3): $2000 \AA$ space $\operatorname{GaAs}\left(T_{g}=600^{\circ} \mathrm{C}\right), 50 \AA$ AlAs $\left(T_{g}=630^{\circ} \mathrm{C}\right), 2$ monolayers (ML) GaAs $\left(T_{g}=530^{\circ} \mathrm{C}\right), 1.7 \mathrm{ML}$ InAs $\left(T_{g}\right.$ $\left.=530{ }^{\circ} \mathrm{C}\right), 30 \mathrm{~s}$ pause, $t_{\text {cap }} \operatorname{GaAs}\left(T_{g}=530^{\circ} \mathrm{C}\right), 30 \mathrm{~s}$ pause, $60 \AA$ AlAs rising $T_{g}$ from 530 to $630{ }^{\circ} \mathrm{C}, 2000 \AA$ space GaAs layer $\left(T_{g}=600^{\circ} \mathrm{C}\right)$ and $300 \AA \mathrm{Al}_{0.4} \mathrm{Ga}_{0.7} \mathrm{As}\left(T_{g}\right.$ $\left.=630^{\circ} \mathrm{C}\right)$. The last layer of QDs $\left(t_{\text {cap }}=50 \AA\right)$ is embedded in AlAs and covered with $300 \AA$ of GaAs.

The PL measurements were done at low temperature (10 $\mathrm{K}$ ) using an $\mathrm{Ar}^{+}$laser tuned at $514.4 \mathrm{~nm}$ and focused on a spot of $\approx 100 \mu \mathrm{m}$. The emitted light is dispersed by a $0.85 \mathrm{~m}$ double spectrometer and detected with a liquid nitrogen cooled Ge detector with a spectral resolution of $0.5 \mathrm{~nm}$. The top layers of sample $\mathrm{E}$ are etched away ${ }^{14}$ at a slow etch rate $(60 \AA / s)$ solution $\left(\mathrm{H}_{3} \mathrm{PO}_{4}: \mathrm{H}_{2} \mathrm{O}_{2}: \mathrm{H}_{2} \mathrm{O}\right)$ to expose successively each layer of QD for carrying out the PL spectroscopy.

Figure 1 shows the PL spectra of samples A, B, and C. As $t_{\text {cap }}$ decreases a blueshift of the ground state emission is observed. For sample A ( $t_{\text {cap }}=50 \AA$ ) and low pump intensity 


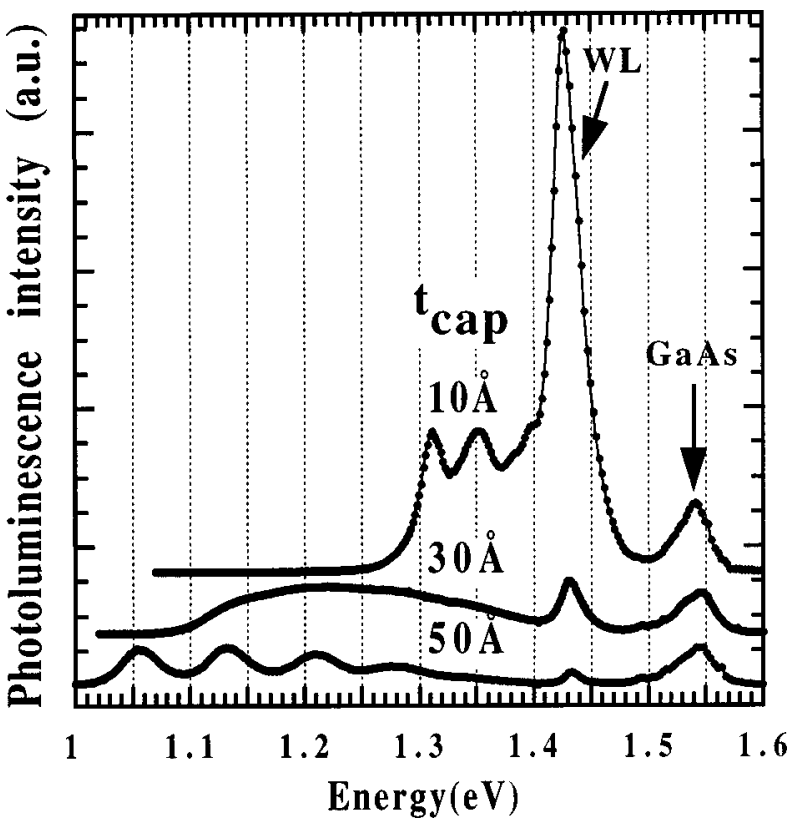

FIG. 1. Photoluminescence spectra taken at $10 \mathrm{~K}$ and $50 \mathrm{~mW} / \mathrm{cm}^{2}$ for samples A, B, and C. The emission from the QDs is blueshifted closer to the WL emission line $(\sim 1.43 \mathrm{eV})$ as $t_{\text {cap }}$ decreases from 50 to $10 \AA$.

$(\sim 1 \mathrm{~mW})$, the spectrum shows a single peak at $1.057 \mathrm{eV}$ (not shown), while at $50 \mathrm{~mW}$, four luminescence peaks equally spaced approximately $75 \mathrm{meV}$ are observed (Fig. 1). When $t_{\text {cap }}$ is reduced to $30 \AA$, the PL spectrum is blueshifted with a significant broadening of the lines that is probably associated with a change in the shape and size of the QDs. This effect has been reproduced in other samples and can also be seen in Fig. 2 for sample E. For sample C ( $t_{\text {cap }}$ $=10 \AA$ ), the emission is further blueshifted. The ground state luminescence is observed at $1.311 \mathrm{eV}$ and the energy difference between higher order recombination lines is reduced to $\sim 42 \mathrm{meV}$.

For all these samples (Fig. 1), the wetting layer PL emission appears at the same energy $(1.43 \mathrm{eV})$ in agreement with an expected constant WL layer thickness. The observed GaAs donor-acceptor pair (DAP) exciton recombination in samples $\mathrm{A}, \mathrm{B}$, and $\mathrm{C}$ appears at $1.55 \mathrm{eV}$ because of the conduction band degeneracy of the heavily doped back contact GaAs layer.

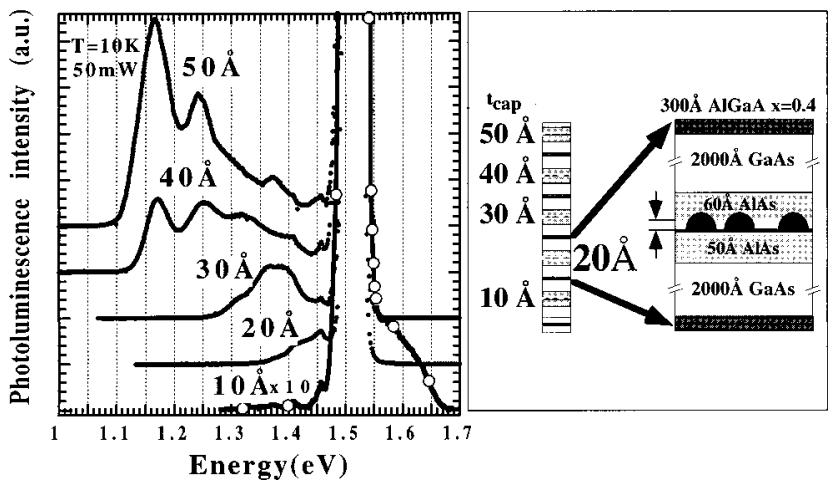

FIG. 2. Quantum dots photoluminescence for sample E $\left(50 \mathrm{~mW} / \mathrm{cm}^{2}, T\right.$ $=10 \mathrm{~K})$. The ground state PL line emission is blueshifted beyond the GaAs exciton emission $(\sim 1.52 \mathrm{eV})$ as $t_{\text {cap }}$ decreases from 50 to $10 \AA$. The spectrum for $t_{\text {cap }}=10 \AA$ is plotted $\times 10$.

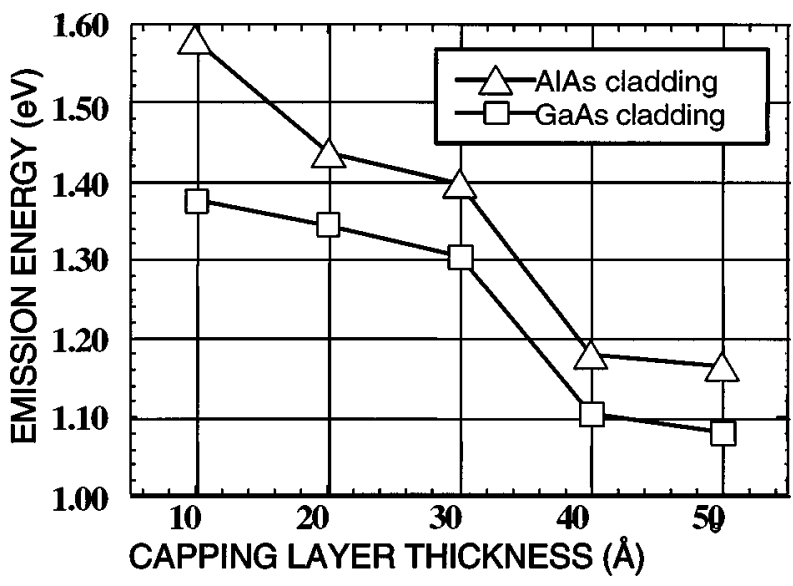

FIG. 3. Quantum dots ground state emission energy $(T=10 \mathrm{~K})$ vs capping layer thickness, $t_{\text {cap }}$, for InAs QDs embedded in AlAs or GaAs cladding layers.

Since covering partially the InAs islands with $t_{\text {cap }}$ reduces their vertical size, it is reasonable to question whether the QDs obtained with $t_{\text {cap }} \approx 10 \AA$ ( $\sim 3$ monolayers $)$ of GaAs still exhibit QDs with 0D confinement characteristics. With increased optical pump power (from 0.1 to $50 \mathrm{~mW} / \mathrm{cm}^{2}$ ), we verified that the electronic state filling effects observed in the normally grown QDs are also seen with these QDs. The higher energy luminescence lines corresponding to the higher excited states intensities are becoming dominant as the pump power increase and the lower subband luminescence is saturated. Confinement in locally $\mathrm{OD}$ areas due to QW monolayer fluctuation can be excluded since the calculated electronic energy splitting due to $\mathrm{InAs} / \mathrm{GaAs} \mathrm{QW}$ monolayer fluctuations are much bigger $(\sim 110 \mathrm{meV})$ than the measured values. We also note that for the sample with the QDs corresponding to $t_{\text {cap }}=10 \AA$, the wetting layer is much more intense. We attribute this to the reduced thickness of the QDs which induces higher energy quantum confined states and a smaller confinement barrier with the GaAs barrier continuum.

When the QDs are embedded in AlAs (sample E) a blueshift is also observed as $t_{\text {cap }}$ decreases (Fig. 2). Compared to samples A, B, C, and D, the ground state PL emission lines are shifted to higher energy values. As in sample $\mathrm{B}$, for $t_{\text {cap }}$ $\approx 30 \AA$, an abrupt change in the energy position and PL line shape is observed. For $t_{\text {cap }} \approx 10 \AA$ the emission is shifted beyond the GaAs free exciton around $1.56 \mathrm{eV}$. As expected from the larger quantum confinement provided by embedding the QDs in AlAs, all the spectra in Fig. 2 are blue shifted from those in Fig. 1.

As we have previously shown, ${ }^{13}$ the vertical and lateral size and shape of the InAs islands and partially covered islands (PCI) are primarily controlled by the thickness $\left(t_{\text {cap }}\right)$ of the GaAs capping layer which is deposited before the islands are completely covered. As seen in Fig. 3, for the QDs, the observed shift in the ground state luminescence and changes in the energy level spacing of the higher excited states luminescence lines are consistent with the previously reported shape changes of the PCI. For $t_{\text {cap }} \approx 20 \AA$, atomic force microscope measurements show InAs PCI which are $\sim 15 \AA$ high and have an approximately ellipsoidal base with dimensions $\sim 1100 \times 700 \AA^{2}$. The total InAs QD vertical size is cense or copyright; see http://apl.aip.org/about/rights_and_permissions 
then $t_{\text {cap }}+15 \AA \approx 35 \AA$. For $t_{\text {cap }} \approx 10 \AA$, we estimate a vertical size of $\sim 25 \AA$ and similar lateral dimensions $(\sim 1100$ $\times 700 \AA^{2}$ ), while those corresponding to $t_{\text {cap }} \approx 50 \AA$ have a thickness of $\sim 70 \AA$ and a diameter of $\approx 200 \AA$.

As we have previously shown intermixing reactions between InAs and AlAs are important and we expect these reactions to induce changes in the QDs composition when capping the InAs islands with AlAs. Thus we cannot estimate quantitatively if the observed blueshifts observed between Figs. 1 and 2 are entirely due to quantum confinement and/or compositional changes or a mixture of the two.

These results clearly show the dramatic effects of the growth kinetics during the transformation of the InAs islands into InAs QDs during the GaAs capping deposition. Both strain relaxation and intermixing of the $\mathrm{Ga}$ or $\mathrm{Al}$ and In will play a role in these shape and size changes of the QDs. The vertical size reduction of the original InAs islands further indicates that $\mathrm{In}$ and $\mathrm{Ga}$ or $\mathrm{Al}$ diffusion is taking place during the partial capping of the islands. Much remains to be done to understand these kinetic effects, however, as we have shown they can be used to tune the QDs electronic structure without having recourse to changing the misfit strain in the system or the WL thickness.

The authors want to acknowledge the financial support of QUEST an NSF-Science and Technology center (DMR No. 91-20007), the Linköping University (POH), the Deut- sche Forschungsgemeinschaft (PJW) and the Spanish Ministry of Education and Science (JMG) for financial support.

${ }^{1}$ G. Madeiros-Ribeiro, F. G. Pikus, P. M. Petroff, and A. L. Efros, Phys. Rev. B 55, 1568 (1997).

${ }^{2}$ S. Raymond, S. Fafard, P. J. Poole, A. Wojs, P. Hawrylak, S. Charbooeau, D. Leonard, R. Leon, P. M. Petroff, and J. L. Merz, Phys. Rev. B 54, 11548 (1996).

${ }^{3}$ H. Drexler, D. Leonard, W. Hansen, J. P. Kotthaus, and P. M. Petroff, Phys. Rev. Lett. 73, 2252 (1994).

${ }^{4}$ M. Grundmann, N. N. Ledentsov, R. Heitz, L. Eckey, J. Christen, J. Bohrer, D. Bimberg, S. S. Ruvimov, P. Werner, U. Richter, J. Heidenreich, V. M. Ustinov, A. Yu. Egorov, A. E. Zhukov, P. S. Kop'ev, and Zh. I. Alverov, Phys. Rev. Lett. 74, 4043 (1995).

${ }^{5}$ S. Sauvage, P. Boucaud, F. H. Julien, J. M. Gerard, and V. Thierry-Mieg, Appl. Phys. Lett. 71, 2785 (1997).

${ }^{6}$ G. Yusa and H. Sakaki, Appl. Phys. Lett. 70, 345 (1997).

${ }^{7}$ R. Leon, S. Fafard, D. Leonard, J. L. Merz, and P. M. Petroff, Appl. Phys. Lett. 67, 521 (1995)

${ }^{8}$ R. Leon, Y. Kim, C. Jagadish, M. Gal, J. Zou, and D. J. H. Cockayne, Appl. Phys. Lett. 69, 1888 (1996); S. Malik, C. Roberts, R. Murray, and M. Pate, ibid. 71, 1987 (1997).

${ }^{9}$ M. Grundmann, O. Stier, and D. Bimberg, Phys. Rev. B 52, 11969 (1995).

${ }^{10}$ K. H. Schmidt, G. Medeiros-Ribeiro, M. Osestreich, P. M. Petroff, and G. H. Döhler, Phys. Rev. B 54, 11346 (1996).

${ }^{11}$ M. A. Cusack, P. R. Briddon, and M. Jaros, Phys. Rev. Lett. 56, 4047 (1997).

${ }^{12}$ G. Medeiros-Ribeiro, D. Leonard, and P. M. Petroff, Appl. Phys. Lett. 66, 1767 (1995).

${ }^{13}$ J. M. Garcia, G. Medeiros-Ribeiro, K. Schmidt, T. Ngo, and P. M. Petroff, Appl. Phys. Lett. 71, 2014 (1997).

${ }^{14}$ K. W. Berryman, S. A. Lyon, and M. Sergev, Appl. Phys. Lett. 70, 1861 (1997). 